\title{
Unidad de propósito Avances de las juventudes y comunidades aliadas al Programa Educación para el Éxito
}

\section{Deborah Robb Taylor}

Más de uno de cada cinco jóvenes que participan en el Programa Educación para el Éxito (EduÉxito) ya es papá o mamá. Es el caso de Shyron Martin, veintiséis años y originario de la comunidad garífuna de Orinoco en la cuenca de Laguna de Perlas. Shyron es padre de dos hijas, una de nueve años y la otra, bebé, de apenas nueve meses.

El joven ha provisto para su joven familia trabajando una pequeña parcela de tubérculos y musáceas que, junto con la pesca artesanal, conforma el modo de subsistencia en esta comunidad litoral. La comida no falta pero el dinero, sí; ha escaseado siempre. Además, dice el joven, "uno tiene su ambición. Quería una vocación para mí, tener un conocimiento, un oficio, algo con qué hacerme una mejor vida."

Así fue cómo, hace un año y medio, a la edad de veinticuatro, conquistó su bachillerato yendo a la escuela sabatina en Orinoco. Y así fue también, que a inicios de este año, dice Shyron, "hice mi esfuerzo para ir a Bluefields a tomar ese taller de electricidad".

El taller a que refiere es uno de los cursos vocacionales que EduÉxito ofrece a jóvenes de dieciséis a veintinueve años, que no estudian ni trabajan - o carecen de un trabajo formal como en este caso-, pero quieren mejorar la calidad de su vida de forma duradera.

Shyron completó la parte técnica del curso ${ }^{1}$ en mayo y tal fue su fortuna, que ese mismo mes se abrió una vacante en la Delegación de la Empresa Nicaragüense de Electricidad (ENEL) en Orinoco. El joven aplicó y con una carta de recomendación de la Coordinación de EduÉxito, ganó el puesto de operador de la planta generadora que abastece su comunidad. Comenzó a trabajar el dieciséis de julio y "para qué —afirma-me gusta mucho; me siento realizado."

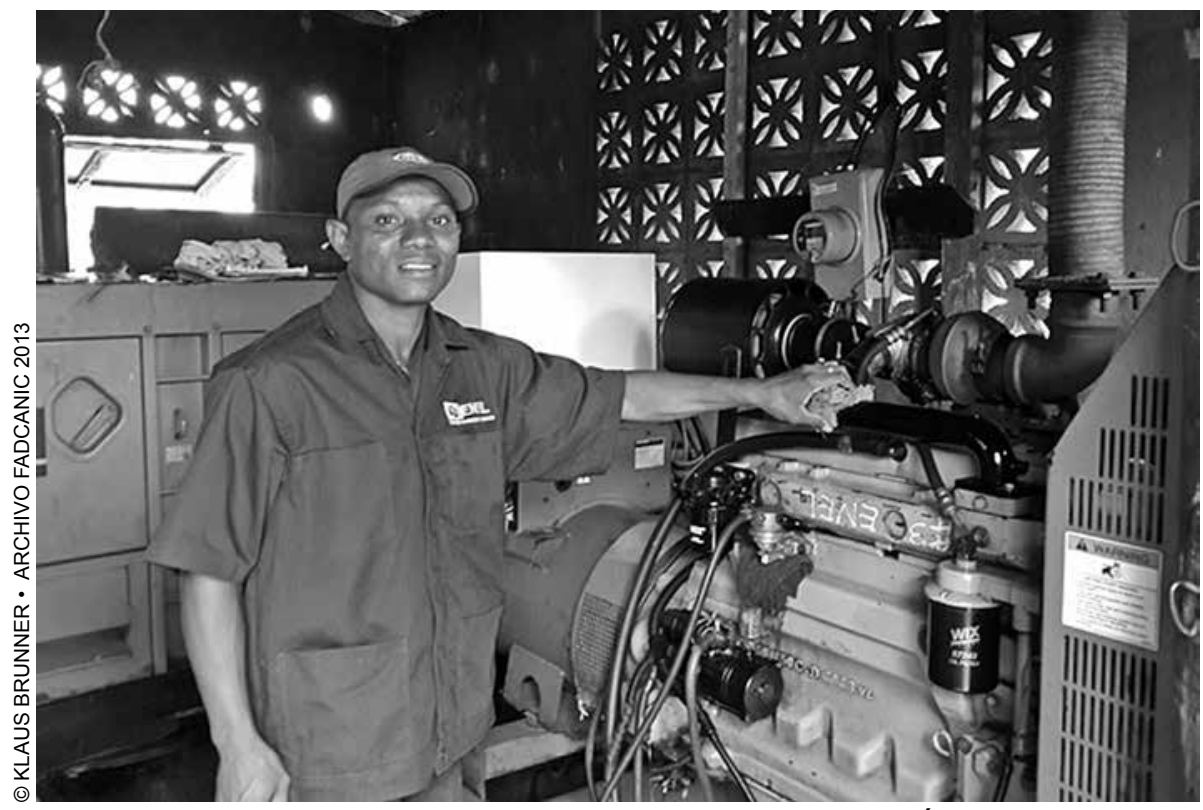

Shyron Martin, egresado de curso de electricidad de EduÉxito, quien ahora labora en la planta de ENEL en la comunidad de Orinoco.

Los cursos vocacionales de EduÉxito también constan de capacitación en iniciativa empresarial y computación básica, además del desarrollo de habilidades para la vida que se da paralelo a la parte técnica. 
Como ésta, aumentan las historias de superación personal de jóvenes que participan en EduÉxito. Desde el 2010, ha atendido a un total de 2,266 adolescentes y jóvenes. De éstos, 1,231 son estudiantes completando estudios de primaria y secundaria mediante becas otorgadas en centros educativos públicos y privados, y 1,035 jóvenes en edad laboral certificados en más de quince carreras técnicas. Entre éstos últimos, casi el diez por ciento, equivalente a 103 jóvenes, ha conseguido un nuevo o mejor empleo acorde con la capacitación adquirida a través de EduÉxito.

Es un logro a ser valorado en un contexto económico regional "poco dinámico" que da pocas oportunidades de trabajo decente a la juventud. Así lo señala el estudio que FADCANIC comisionó en el 2013 sobre mercado laboral juvenil en la $\mathrm{RACS}^{2}$, una de las cinco investigaciones que contiene esta edición especial de Wani.

En su conjunto, estos estudios abarcan información muy valiosa sobre un gran rango de temas y preocupaciones que afectan de forma específica a la niñez y juventud indígena, mestiza y afrodescendiente de los municipios de intervención de EduÉxito. Cada estudio corresponde a una o más áreas de incidencia del Programa, el cual viene orientando y refinando sus abordajes según los hallazgos y las recomendaciones pertinentes de las investigaciones.

\section{Perspectivas estratégicas}

Por ejemplo, el mismo estudio sobre el mercado laboral apunta a la necesidad de forjar alianzas alrededor de proyectos estratégicos dirigidos a la promoción del empleo juvenil en sectores productivos clave. La recomendación casa con la construcción de alianzas públicas y privadas, que es uno de los pilares de acción de EduÉxito; y a este fin, el programa ha iniciado conversaciones este año con empresas nacionales como la Nicaragua Machinery Company.

La NIMAC-CAT, como es conocida, facilita cursos en operación y mantenimiento de maquinaria pesada en coordinación con la Fundación Samuel, que atiende a jóvenes de escasos recursos en su centro de capacitación técnica ubicada en las afueras de Managua. USAID gestionó y participa de este acercamiento entre EduÉxito y NIMAC-CAT, que explora la posibilidad de replicar el currículo de la empresa en la región Caribe, incluyendo sus cursos para técnicos de servicios en motores diesel y en sistemas eléctricos e hidráulicos.

La viabilidad de esto depende, entre otras cosas, de que se disponga de las condiciones necesarias para dictar cada curso, incluyendo salas de entrenamiento multimedia, talleres mecánicos y áreas externas para realizar las prácticas con la maquinaria. Esto se va a seguir explorando en el terreno con visitas próximas de NIMAC-CAT al Centro de Educación Ambiental y Agroforestal (CEAA) que FADCANIC opera en Wawashang, en el municipio de Laguna de Perlas.

El interés estratégico, en todos los casos, está en las perspectivas laborales y de negocios que se vislumbran junto a clientes actuales de la NIMAC, como la Cukra Development Corporation, que cultiva y procesa la palma africana en Kukra Hill, y otras empresas potenciales que surjan de proyectos del porte de la presa hidroeléctrica de Tumarín, en el municipio de la Cruz de Río Grande, la carretera de Nueva Guinea a Bluefields en curso, y el canal interoceánico que según últimas declaraciones del gobierno, cruzará el territorio nacional de este a oeste, uniendo los ríos Brito, en el departamento de Rivas, y Punta Gorda, en la RACS.

\section{Personalización de la enseñanza}

Las investigaciones también están ayudando a que el Programa mantenga su enfoque sobre sus métodos pedagógicos. El estudio de línea base de Educación para el Éxito $2013-2017^{3}$ fue realizada por el consultor de la American Institutes for Research Joel Zamora. Éste encontró que la mitad de adolescentes y jóvenes que están en EduÉxito, "no entiende" o sólo "más o menos entiende" las explicaciones que le brindan docentes o instructores en sus centros de estudio.

Es una situación que tiene que ver en parte con la predominancia de la enseñanza en el idioma español entre jóvenes con distintas lenguas maternas; y en esta medida, el Programa busca, siempre que sea posible, que la instrucción se dé en el idioma local. Así, por ejemplo, el curso de electricidad que hizo Shyron Martin en Bluefields fue impartido por un electricista industrial de ascendencia

Fonseca, Roberto. 2014. “Hacia una inserción laboral efectiva en la RACS” Wani, 69, pp. en la página 31 
garífuna, cuya lengua madre es el inglés creole, como el propio Shyron, quien valora que "realmente ayudó mucho que le pudiese preguntar las cosas en inglés, porque a veces no nos alcanzaban las palabras en español para tantas cosas técnicas".

Sobre la cuestión del grado de comprensión y calidad del aprendizaje, que tiene que ver igualmente con cuestiones de confianza y de personalización de la enseñanza, EduÉxito también viene fortaleciendo las tutorías o sesiones de refuerzo escolar para sus becarios y becarias de primaria y secundaria.

Estas becas para la educación formal de calidad son dirigidas a niños, niñas y adolescentes de diez a diecinueve años. El programa capta chavalas y chavalos con riesgo de exclusión o abandono escolar y les facilita tres años de atención integral para que puedan completar la primaria o el ciclo básico de la secundaria. En esta modalidad, EduÉxito también oferta la posibilidad de cursar una carrera de técnico básico en el CEAA en Wawashang, o en uno de los establecimientos de INATEC en Bluefields o Corn Island.

Dada la prevalencia de las dificultades de aprendizaje, las tutorías de afterschool, como EduÉxito denomina sus clases de refuerzo académico, se vuelven vitales al buen desempeño escolar. Estas tutorías son impartidas, por lo general, dos veces a la semana por docentes seleccionados, que atienden a los y las becarias de primaria, y por estudiantes sobresalientes, que atienden a los y las de secundaria. Focalizan su atención en quienes están sacando notas bajas en inglés, español y matemáticas, pero el refuerzo se puede extender a cualquier otra materia en que se esté teniendo dificultad.

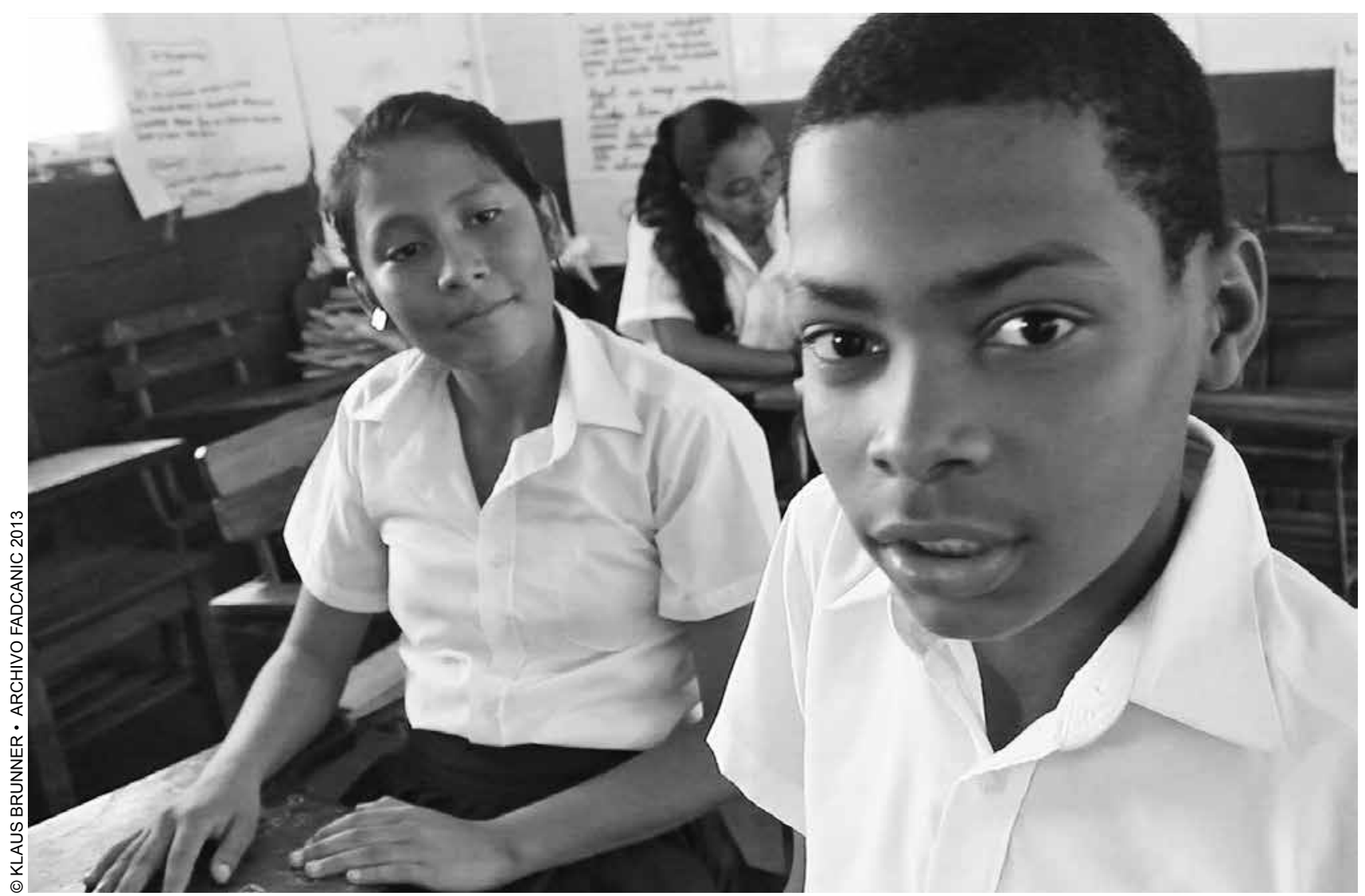

Becarios de EduÉxito Junior Orozco y Scarlett Ellis, ambos de Kukra Hill.

La beca de EduÉxito cubre el suministro de uniformes, materiales escolares, costo de matrícula y mensualidades en el caso de los centros privados, y costos de transporte y de alimentación cuando se estudia fuera de la localidad de origen del estudiante. La beca de EduÉxito también incluye servicios gratuitos de consejería psicosocial, referencias a otros servicios especializados de salud y bienestar integral y educación en habilidades para la vida mediante participación en prácticas deportivas, artísticas y musicales. Los becarios regulares gozan estos beneficios por tres años. 
Asimismo, la atención no se limita a las sesiones de grupo programadas en el centro escolar sino que, también, abarca otras actividades de aprendizaje. Como atestigua Junior Orozco quien cursa la secundaria en el Colegio Hugo Heinze de Kukra Hill: "lo que me gusta es que mi monitora me da el repaso siempre que la busco; me ayuda también con las tareas, no tiene que ser sólo a la hora de la tutoría". Durante el primer corte evaluativo este año, Junior sacó 59 en matemáticas. Ya para el segundo, con la ayuda de su joven tutora, Scarlett Ellis, su nota subió a un más que decente: 82 .

A nivel del Programa, el índice de promoción de un año académico al otro ha sido de $98.5 \%$ para un total de 910 niños, niñas y adolescentes que cursaron primaria y secundaria con el apoyo de EduÉxito durante el período 2010-2013. En 2013 incluso, EduÉxito concluyó el año lectivo regular con apenas 90\% de sus estudiantes aprobando todas sus asignaturas. Aún estando debajo del promedio del Programa, esta tasa de promoción ya superaba en siete puntos porcentuales al promedio regional de $83 \%$ registrado por el MINED para el sistema educativo como un todo. Entretanto, la promoción 2013 de EduÉxito llegó a elevarse a 99\% tras un mes intensivo de clases de refuerzo para alumnos y alumnas que habían reprobado una o más materias, recuperadas después en los exámenes de reparación.

\section{Tranquilidad, confianza y persistencia}

Una de las particularidades del programa de tutoría académica de EduÉxito, es que los estudiantes que sirven de monitores también son seleccionados entre el estudiantado de más bajos recursos económicos y, en su calidad de tutores de afterschool, pasan a recibir los beneficios integrales de la beca de EduÉxito por un período de un año ${ }^{4}$. De esta forma, el Programa ayuda a crear entre ellos la vocación de servicio y les da la oportunidad de acceder a experiencias que de otra manera no estarían a su alcance.

Así lo señala, por ejemplo, Perla Hernández, que se integró a EduÉxito en 2014. Perla tiene quince años, estudia el noveno grado en el Colegio 30 de Octubre, de Bluefields, y considera que es "una gran responsabilidad que me hayan elegido. Tengo que ayudar a otros estudiantes y tampoco me puedo descuidar de mis estudios, pero organizándome con el tiempo sé que lo voy a poder controlar". Al mismo tiempo, agrega la nueva tutora, "me parece que es algo en que me puedo divertir, aprender cosas nuevas sobre la vida y relacionarme con otras personas porque yo no salgo de mi casa".

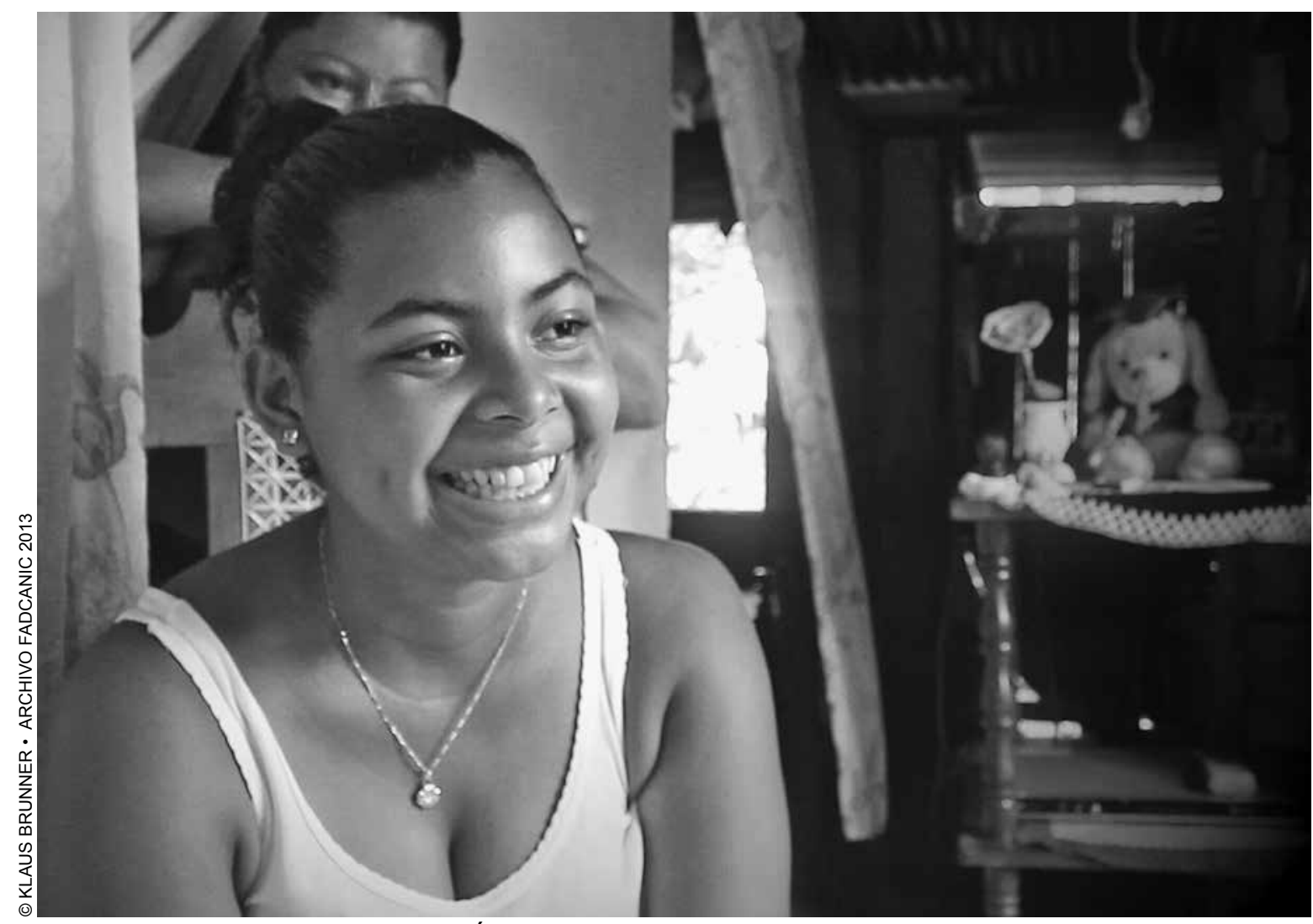

Perla Hernández, Becaria de EduÉxito en el municipio de Bluefields. 
En efecto, la vida de Perla ha sido "de la casa a la escuela y de la escuela a la casa" y desde muy temprana edad se ha tenido que dedicar al lavado de carros, que es el negocio con el que se mantiene la familia. Ahora la joven también consigue ocupar un par de tardes a la semana para ir a los ensayos del grupo coral al que se ha unido en la Casa de Adolescentes de EduÉxito y los viernes atiende sesiones lúdicas donde, según lista la joven, "jugamos, nos divertimos todos juntos, nos relacionamos, compartimos cómo hemos estado y hablamos sobre cómo nos sentimos, por si tenemos algún problema, tengamos la confianza de decirlo para que alguien nos pueda ayudar".

Para EduÉxito es terriblemente importante asegurar estos espacios de recreación sana pero también de tranquilidad, confianza y mutua ayuda a toda su población beneficiaria. Como Perla, casi un tercio de adolescentes y jóvenes en el Programa tiene que compaginar el trabajo y el estudio, otra cantidad está regresando a sus estudios después de uno o más años de haber abandonado la escuela y, más allá de la pobreza generalizada, su situación familiar tiende a ser atravesada por tragedias, conflictos, abusos y abandonos.

Es por esto que EduÉxito se define como un Programa de crecimiento y desarrollo personal, Programa, que no sólo da becas escolares a niños y adolescentes, o capacitación para el trabajo a jóvenes en edad laboral, sino que también les asegura servicios de consejería psicosocial, capacita a sus progenitores en cómo mejorar las relaciones familiares, capacita a sus docentes en estrategias de aprendizaje emocional y social, y desarrolla sus habilidades para la vida, de tal forma que puedan resistir a los riesgos en su entorno e insertarse de una forma más efectiva a los procesos educativos, sociales y productivos.

En este sentido, el Programa está poniendo gran énfasis en enseñar a estos jóvenes a no darse por vencidos. Johnny Escobar, de Tasbapauni, ejemplifica esta cualidad. Johnny tuvo que dejar de estudiar en 2011 cuando estaba en el quinto grado por un accidente que casi le corta un pie a la mitad. Tenía trece años en la época y su recuperación fue demorada, "ya no pude estudiar ese primer año, el siguiente también lo volví a perder y ya iba otro año que tampoco pude comenzarlo, entonces estaba con miedo de que también lo iba a perder".

Felizmente, ese mismo semestre, en agosto de 2013, EduÉxito abrió su primer programa de primaria acelerada en Tasbapauni. Johnny se matriculó y a través de ella se graduó del sexto grado el siete de febrero de 2014. A la semana, cuando abrió el curso lectivo, el joven ya estaba cursando su primer año de secundaria. Cuando le salió el primer boletín, este abril pasado, el muchacho no estaba muy contento consigo mismo, pues, según dijo, perdió unos apuntes y se sacó una nota roja. Varios compañeros y compañeras que hicieron el curso de primaria acelerada con él, ya habían desistido de continuar la secundaria. "Pero yo no voy a dejar de ir a clases," asegura Johnny, "voy a andar con mi diploma de secundaria porque andar por allí de vago no lleva a nada".

\section{La primaria acelerada de EduÉxito}

Como se desprende, el programa de primaria acelerada es la opción de EduÉxito para estudiantes de extra-edad que aún no han concluido la primaria pero tienen deseos de continuar sus estudios. Según hallazgos del estudio de Ethel Martínez sobre Vulnerabilidad socioeducativa y económica de adolescentes y jóvenes en cinco municipios de la $R A A S,{ }^{5} 14 \%$ de adolescentes y jóvenes de quince a veinticuatro años se encuentran en esta condición en los municipios de intervención de EduÉxito. Mediante la primaria acelerada de EduÉxito podrían cubrir, en seis meses, los contenidos de matemáticas, lengua y literatura (español) e inglés como segundo idioma, correspondientes al cuarto, quinto y sexto grado de la primaria.

Durante ese mismo período cubrirían también el currículo de habilidades para la vida, de EduÉxito, que desarrolla la autoestima y las capacidades de comunicación asertiva y de toma de decisiones y elaboración de planes de vida, obteniendo así el diploma de sexto grado convalidado por el MINED. El modelo aún está siendo validado, pero no hay como exagerar el éxito que tuvo su implementación el año pasado en Tasbapauni. La maestra Katia Molina, una docente sustituta de veintinueve años, que asumió el desafío de liderar esta primera experiencia, lo describe así:

"El objetivo era conseguir que estos jóvenes desarrollasen su capacidad de lecto-escritura en los dos idiomas y sus habilidades numéricas a un nivel de sexto grado, porque, dada su edad, la primaria no iba a poder aceptarlos al año siguiente. Estamos hablando de chavalos de hasta veinte años el mayor. El colegio abrió una clase de nivelación para algunos de ellos, pero no estaban 
avanzando. Había necesidad de alguien que se dedicase exclusivamente a ellos, que les escuchase, que oyese sus dificultades, que consiguiese que trabajasen de forma conjunta. Estos eran chavalos y chavalas que podían haberse matriculado para estudiar pero no tenían un hábito de estudio. Llegaban y salían del colegio cuando querían. Venían de todos los niveles, algunos nomás hasta el tercer grado habían llegado.

Comenzamos con 18, entre ellos 15 varones y tres mujeres. En los seis meses perdimos solamente a uno. Al inicio fue extremamente difícil, porque a veces había más gente fuera de la sala de aula que adentro; la clase se había vuelto una curiosidad y no faltaban quienes se asomasen sólo para provocar. Los muchachos se avergonzaban y había que decirles con mucho amor y con mucha delicadeza "tenemos que mostrarle a cualquiera allá afuera, que éste es nuestro lugar y queremos que lo respeten".

Con el tiempo las dificultades desaparecieron. Los miembros del CAYAC ${ }^{6}$ hicieron su parte. Llegaban a acompañar las clases para prevenir los disturbios. Cuando algún alumno faltaba más de un día, iban a hablarle a él o a la familia. Si acostumbraban llegar tarde, hacían lo mismo; iban a recordarle al estudiante que ya era hora de ir a la clase.

La escuela hizo su parte. Cedió el espacio para estos muchachos y muchachas en su horario vespertino permitiendo que se sintiesen como parte de una escuela diurna regular y no como un nocturno o sabatino. La directora supervisaba, aplicaba las comprobaciones de lectura y ante el desempeño de la clase en las pruebas autorizó su promoción. Educación para el Éxito hizo su parte. El programa asumió mi salario como docente, fui capacitada y apoyada con otras personas que llegaban a facilitar las clases de habilidades para la vida y jamás faltaron los materiales didácticos.
Con el tiempo, algunos papás y mamás también fueron haciendo su parte, asistiendo a las reuniones escolares y acompañando el desempeño de sus hijos. Y los que estudiantes que no tenían mamá o papá, como era el caso especialmente de las tres muchachas, sencillamente se hicieron de la cabeza que esto es lo que querían: concluir sus estudios de primaria. Así lo hicieron y lo hicieron bien".

Para la graduación en febrero, el liderazgo comunitario de Tasbapauni, que fue el que había solicitado que EduÉxito realizase el curso de primaria acelerada en su comunidad, volvió a hacer su parte asegurando una ceremonia de gala presidida por la vice alcaldesa del municipio y la delegada municipal del MINED.

De los 17 que se graduaron ese día, ocho continúan la secundaria incluyendo uno de los jóvenes que se transfirió a Corn Island donde fue aceptado en una institución de enseñanza media sin ninguna dificultad. "Es el poder de un diploma," enfatiza la integrante del CAYAC de Tasbapauni, Quinny Julias de 22 años, quien afirma también que "ya estamos pensando cómo involucrar a quienes que no siguieron sus estudios, en alguna clase de computación, si se compone la electricidad ${ }^{7}$, o en algún curso vocacional que les llame la atención".

\section{Grados singulares de apropiación del programa}

Para la coordinadora del Programa Educación para el Éxito, Hazel Wilson, las sinergias que se generaron alrededor del programa de primaria acelerada son evidencia de "una capacidad propositiva que va creciendo en prácticamente todas las comunidades en las que estamos trabajando". Antes FADCANIC llegaba con una iniciativa y tenía que hacer todo, indica Wilson, "pero ahora no; ahora el liderazgo comunitario juzga sí se trata de algo que le va aportar a su bienestar y a su desarrollo y en esa medida se posiciona como un aliado de estatura que cuestiona, demanda y sobretodo, propone".

También sostiene y expande. En Corn Island este año, Educación para el Éxito equipó a 91 estudiantes

6 Comités Comunitarios de Asesoría a la Juventud en Situación de Riesgo. Son estructuras integradas por padres y madres de familia, docentes y líderes comunitarios que, usando criterios e instrumentos de selección estandarizados, identifican y seleccionan a los y las beneficiarias del proyecto y acompañan a estos jóvenes y a sus familias durante toda su trayectoria en el Programa.

Tasbapauni se ha conectado a la red nacional de electricidad, pero el suministro eléctrico todavía es altamente irregular. 


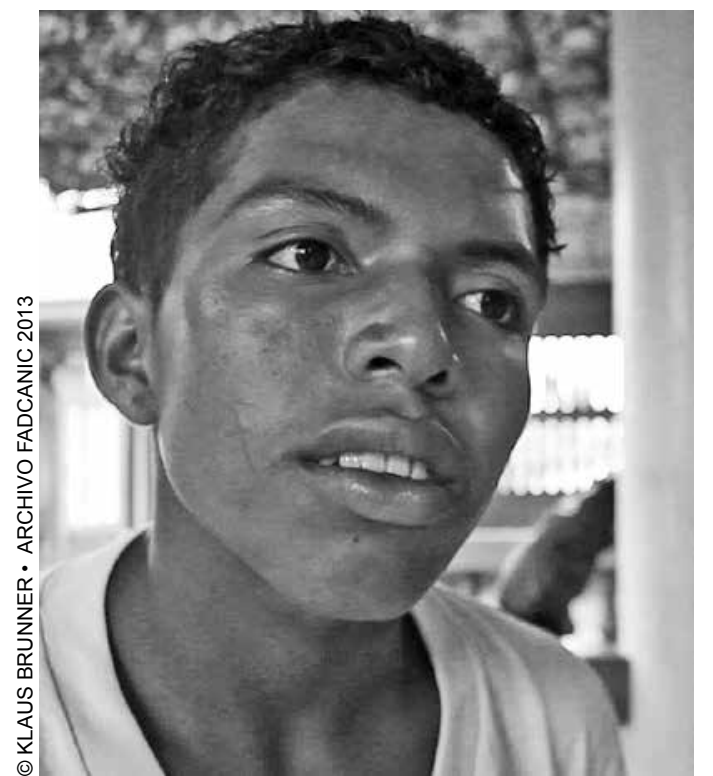

Johnny Escobar, egresado del curso de primaria acelarada de EduÉxito en la comunidad de Tasbapauni.

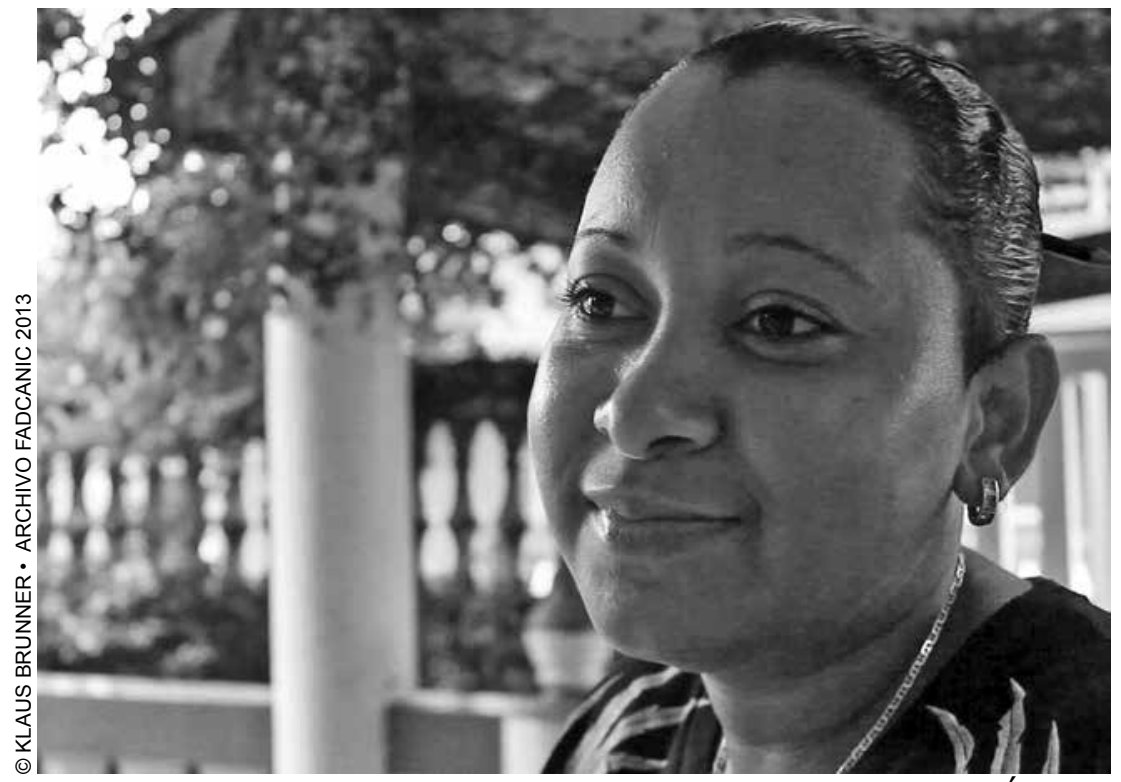

Katia Molina, docente del curso de primaria acelerada de EduÉxito de primaria y secundaria con el paquete completo de materiales escolares que requieren para el curso lectivo. El CAYAC de Little Island equipó por cuenta propia a otros 104 alumnos y alumnas. En ánimo similar, la alcaldía de Corn Island donó a la Casa de Jóvenes de EduÉxito una cocina para contribuir a las ventas que hace el CAYAC para recaudar fondos. El arrendador del edificio donde funciona la Casa de Jóvenes no se ha quedado atrás, él le cedió al programa dos mantenedoras para el mismo propósito.

En Laguna de Perlas, Educación para el Éxito también cuenta con el apoyo de la alcaldía municipal que se traduce, por ejemplo, en la inserción de jóvenes que han egresado de cursos vocacionales del programa a los cursos de computación que la alcaldía está comenzando a impartir en la sede del municipio. Con la Comuna de Orinoco, se plantean nuevos cursos de corte y confección, abiertos a jóvenes de toda la cuenca, para aprovechar maquinas de costura industrial propias de la comunidad. En Bluefields, la Secretaría Regional de la Juventud (SEREJUVE) ya ha alcanzado acuerdos preliminares con EduÉxito que están facilitando el acceso de los integrantes del programa los estudios de producción y transmisión de la emisora radial Bluefields Estéreo y al centro de tecnologías multimedia que la SEREJUVE está instalando con el apoyo de entidad de cooperación alemana GIZ.

En la Desembocadura de la Cruz de Rio Grande también se ha visto un grado muy singular de apropiación del Programa Educación para el Éxito. Aquí, los CAYACs tienden a ser integrados en mayor número por autoridades territoriales y comunitarias entre las cuales los miembros del Consejo de Ancianos. Aquí también, los acuerdos de cooperación con la alcaldía municipal incluyen, desde el 2013 (cuando el municipio ingresó a EduÉxito), cesión gratuita del espacio para la oficina sede del Programa y facilidades de cuido de bebés de mujeres jóvenes que asisten a los cursos vocacionales ${ }^{8}$.

Desembocadura también tiene la singularidad de que padres y madres participan de forma activa de todas las actividades de EduÉxito, lo cual sigue siendo un reto en muchas comunidades. Edgar Santiago Knight, de veintiséis años y promotor municipal de EduÉxito, aduce que se debe tanto al compromiso que tienen sus coterráneos con el futuro de sus comunidades, como a los efectos de las labores educativas del Programa.

Este beneficio se extiende ahora a todas las jóvenes con bebés menores de dos años que integren cursos vocacionales de EduÉxito en los cinco municipios. 


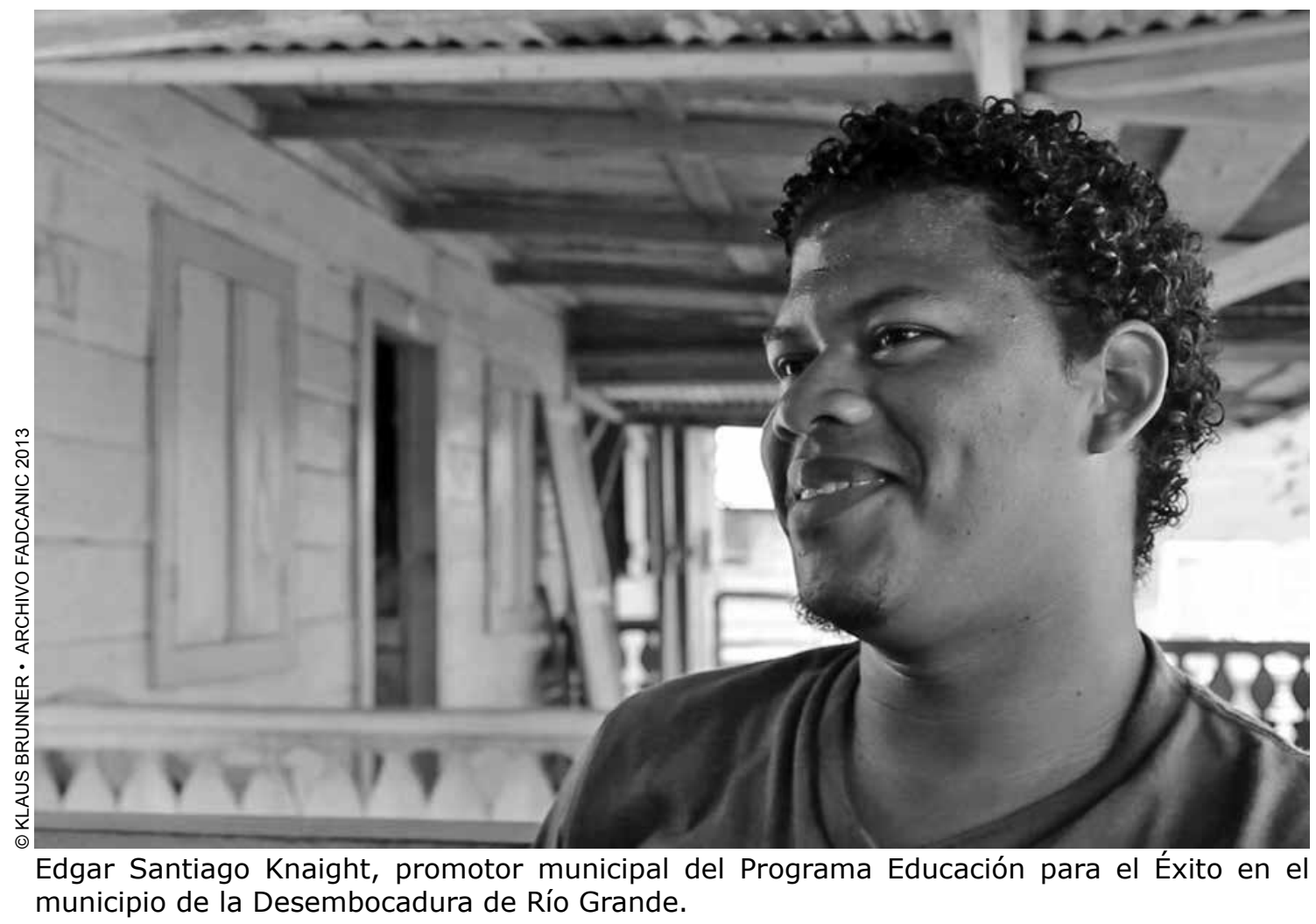

Dice Edgar:

"Tenemos una honda preocupación por la salud, la seguridad y el futuro de nuestra comunidad y de nuestra juventud, entonces no tenemos problemas para que las personas vengan a las reuniones $y$ participen de los acuerdos. Este año, por ejemplo, la dirección de la secundaria en Karawala pidió ayuda porque sentía que los alumnos se estaban alborotando mucho. Ahora padres y madres están tomando turnos para visitar las salas de aula y la paz se ha recuperado. Son los mismos padres que están asistiendo a los círculos de good parenting de Educación para el Éxito, los mismos docentes que van a los talleres de aprendizaje emocional y social, los mismos voluntarios que están aprendiendo a conducir las clases de habilidades para la vida, y los mismos líderes que están aprendiendo a velar por la conducta ética de las personas que están trabajando con los jóvenes. Entonces, claro que se comienza a ver el cambio".

\section{La consejería de EduÉxito}

Noelia Powell, docente de secundaria y coordinadora del CAYAC y de la Comisión Municipal de la Niñez y
Adolescencia de Kukra Hill, describe ese cambio, "Los alumnos que pasan por este programa no sólo mejoran en el aspecto de la calificación o de la rebeldía. Mejoran en la convivencia, en lo social, en lo más personal de ellos porque aquí consiguen sacar todas aquellas cosas que frustran al adolescente."

Según Powell, es un cambio que comienza con el propio docente. "No es como cuando yo tengo un bebé, le cambio el pañal, le pongo la pacha, lo acomodo y pronto," dice la docente. "El adolescente es un poco más delicado que eso, no le puedo tratar con rebeldía ni con demasiada condescendencia. Tengo que saber la manera de llegarle a ese adolescente y es aquí donde entran las virtudes de la consejería de Educación para el Éxito. Porque el asunto no es sólo de la cabeza, también hay que aprender a sentir".

La docente también destaca que la virtud principal de la consejería de EduÉxito es que "no atiende sólo al niño o al adolescente o al joven "problema". Está atendiendo también a la familia, ya sea mamá, papá, abuela. Entonces uno comienza a ver ese cambio de mentalidad del que pensaba que su responsabilidad era mandar a sus hijos a la escuela y nada más, o de quien ya ni cuenta se daba de que todo aquello que había odiado de su propia niñez o su actual estación en la vida, lo estaba descontando sobre 
sus propios hijos. No es de la noche a la mañana, pero prestan más atención, se fijan más en lo que dicen y uno ve el cambio de los chavalos en las actividades, llegan más, llegan más temprano, participan y se abren más”.

La consejería también toca a los miembros de CAYACs, "que acompañamos todas las actividades de estos jóvenes en las tutorías, en las mentorías de pares ${ }^{9}$, en los intercambios aquí mismo y cuando van a otras comunidades. Si es la juventud que va con toda la leche y uno como adulto que tiene la responsabilidad, tiene que entrenarse también para estar en condición de manejarse con tranquilidad y con prudencia. Somos los espejos de estos chavalos y eso lo tenemos clarísimo", finaliza Powell.

\section{Otra raza de líderes comunitarios}

A propósito de cómo se manejan grupos adolescentes y jóvenes que se movilizan en actividades a veces a los cientos, Hazel Wilson también ve indicios de cambio de comportamiento de grupo bastante positivos. "Durante el programa de revitalización cultural de 2013 en Laguna de Perlas, llevamos a más de 50 jóvenes de distintas comunidades de la cuenca y de otros municipios que hospedamos en hoteles durante todo el mes. En uno de los hoteles tuvimos que pagar hasta lavamanos y abanicos rotos."
Volvió a suceder durante las actividades de la Semana de la Juventud de agosto 2013 en Bluefields. "Desde las quejas entre los propios jóvenes sobre a quién le robaron qué, hasta las de los dueños de hotel sobre los disturbios que le causaban a los otros clientes; era una situación en que se tenía que intervenir," relata Wilson. ¿Y la intervención? Seminarios de pre-salida para cada delegación de jóvenes antes de cada excursión para definir las expectativas del grupo, dirimir dudas y consensuar las reglas de conducta. En casos de estancias prolongadas fuera de la propia comunidad, el Programa también ha pasado a disponer de un consejero o consejera en los locales de alojamiento para apoyar los servicios de chaperone que realizan padres, madres y miembros de CAYAC que acompañan las delegaciones.

Las medidas están funcionando. "En enero este año volvimos a movilizar un gran grupo de jóvenes a Laguna de Perlas y no tuvimos que pagar ni un plato roto. Los que llegaron al curso de producción de radio y TV en Bluefields, igual, ni una sola queja de ningún hotel," afirma Wilson.

La coordinadora del Programa también destaca que intervenciones como las que se han aplicado en este caso, no fueron ideas que emanaron del equipo técnico de FADCANIC sino de ese cuerpo más extendido de

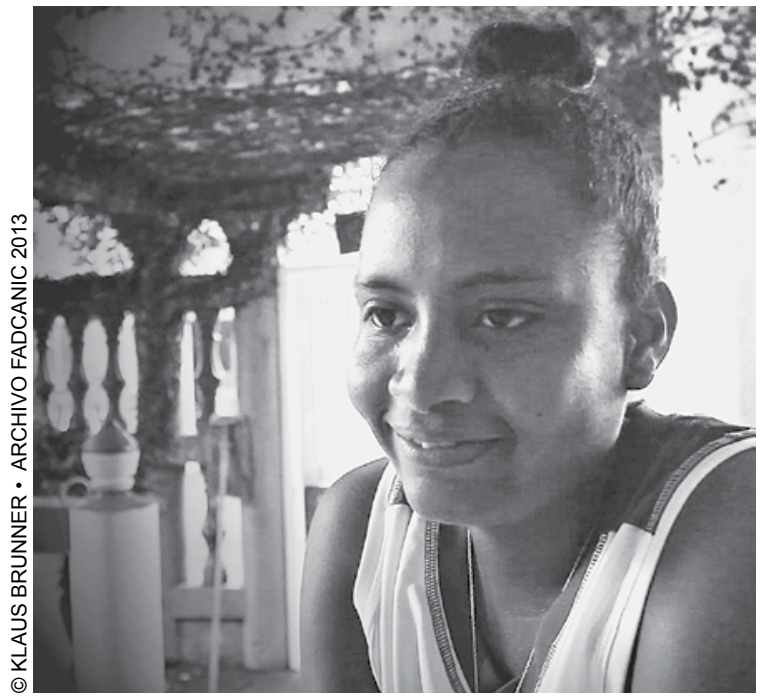

Quinny Julias, miembro del CAYAC de Tasbapauni.

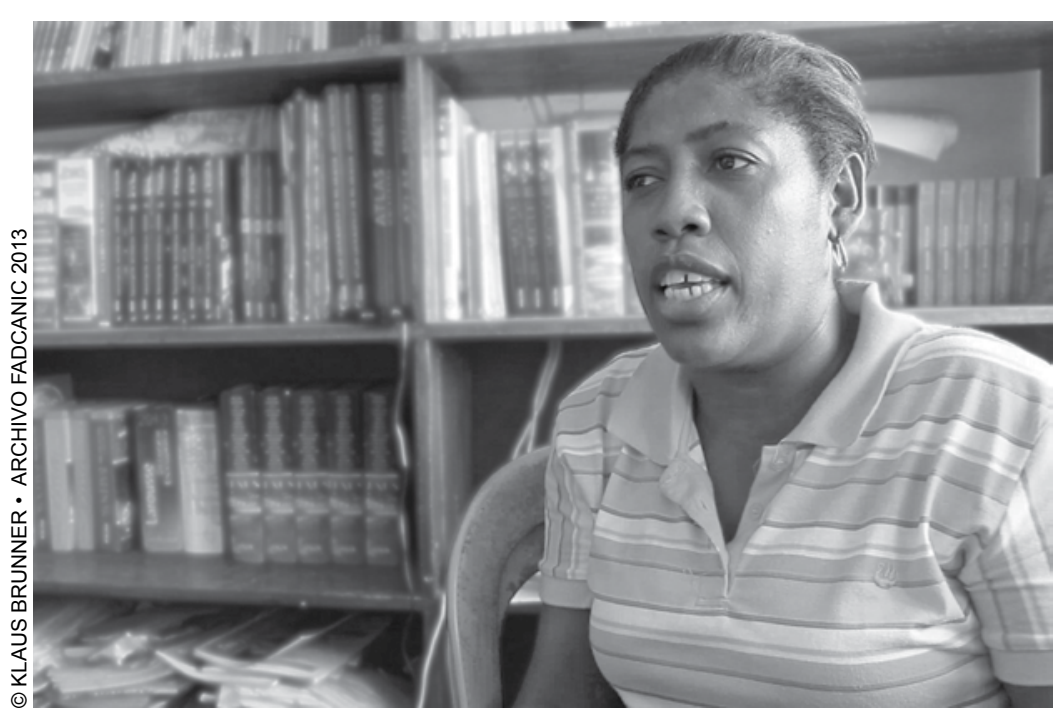

Noelia Powell, Coordinadora del CAYAC de Kukra Hill.

\footnotetext{
9 EduÉxito también ha capacitado a 241 adolescentes y jóvenes del programa para conducir el currículo de habilidades para la vida e impartir charlas regulares con otros jóvenes dentro y fuera del programa, sobre temas vinculados a los riesgos y a las oportunidades sociales en su comunidad.
} 
colaboradores del programa EduÉxito que al mes de julio de 2014 contaba 171 voluntarios y voluntarias, entre los cuales 136 miembros activos de CAYAC y 35 instructores de distintos géneros y disciplinas artísticas, musicales y deportivas que ahora hacen parte del día a día adolescentes y jóvenes que participan en el Programa.

Como reitera Noelia Powell, "Me gusta como estamos trabajando; EduÉxito no es el padrino, el papá, el Tarzán, no. Él nos da las herramientas y nosotros lo echamos a andar."

Como responde Quinny Julias, “¿Qué si yo creo que hay futuro para los jóvenes en mi comunidad? Antes uno les oía decir, ‘¿yo que hago? Aquí no hay nada para hacer'. Ahora uno oye, 'voy a montar un salón de belleza, voy a abrir un puesto de reparación de celulares, voy a hacer una sorbetería de frutas tropicales' y es bonito ser parte de todo eso."
Y como apunta Hazel Wilson:

"Aqui hay que reconocer que a pesar de la complejidad de la región y todos los desafios que desnudamos cuando profundizamos en los riesgos que caracterizan nuestra realidad, estamos frente a una nueva raza de líderes comunitarios, que entre otras cosas son cada más jóvenes y cada vez menos obcecados con escándalos y disputas intestinas. Lo vemos en Tasbapauni donde la comunidad se fincó, la vieja guardia cedió y con ella se fue toda una era de conflictos por la tierra. De Awal Tara, que es el territorio de la Desembocadura, ya ni hablemos, hasta su vocabulario es diferente, no hablan de beneficiarios hablan de sociedad. En Orinoco, Kahkabila, Laguna de Perlas, es igual, son jóvenes y son electos por las comunidades. Kukra Hill y Little Island dan lecciones de organización comunitaria, El Bluff y Rama Cay hasta aqui están llegando los cambios y las oportunidades que necesitamos visibilizar".

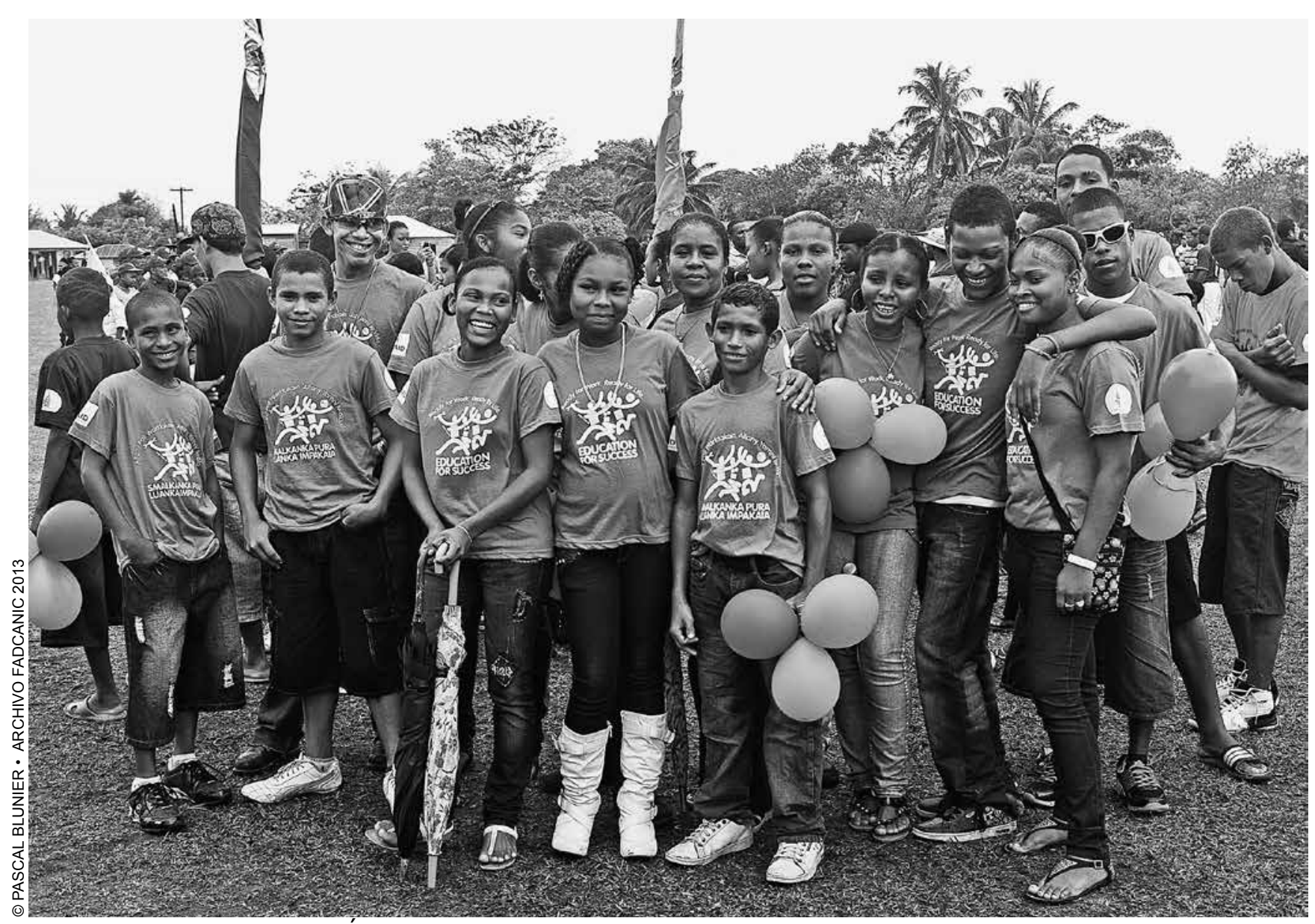

Becarios y becarias de EduÉxito junto a la coordinadora del Programa, Hazel Wilson, en Laguna de Perlas. 